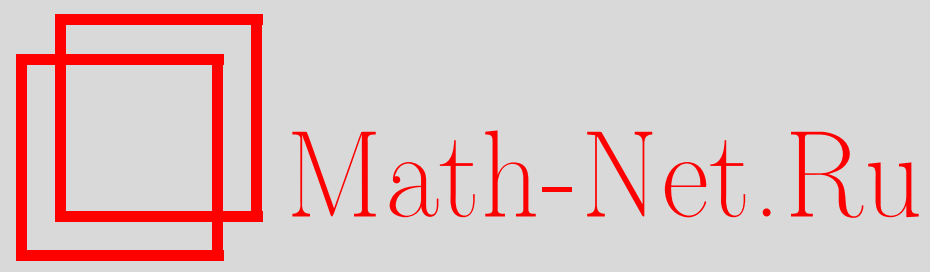

Е. Б. Кузнецов, Преобразование уравнений с запаздывающим аргументом к наилучшему аргументу, Матем. заметкu, 1998, том 63, выпуск 1, 62-68

DOI: https://doi.org/10.4213/mzm1248

Использование Общероссийского математического портала Math-Net.Ru подразумевает, что вы прочитали и согласны с пользовательским соглашением http://www . mathnet.ru/rus/agreement

Параметры загрузки:

IP : 54.196 .121 .252

26 апреля 2023 г., 04:27:03

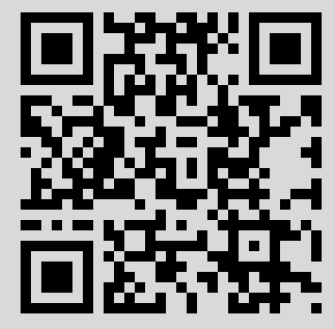




\section{ПРЕОБРАЗОВАНИЕ УРАВНЕНИЙ С ЗАПАЗДЫВАЮШИМ АРГУМЕНТОМ К НАИЛУЧШЕМУ АРГУМЕНТУ}

\section{Е.Б. Кузнецов}

$\mathrm{C}$ точки зрения метода продолжения решения по параметру исследуется вопрос о выборе наилучшего аргумента в задаче Коши для системы обыкновенных дифференциальных уравнений с запаздывающим аргументом. Устанавливается, что наилучшим аргументом, обеспечивающим наилучшую обусловленность системы уравнений продолжения, является длина дуги, отсчитываемая вдоль интегральной кривой задачи. Получено преобразование задачи Коши к наилучшему аргументу.

Библиография: 9 названий.

1. Введение. Рассмотрим решение задачи Коши для обыкновенного дифференциального уравнения с запаздьвающим аргументом [1]:

$$
\begin{gathered}
\frac{d y}{d t}=f(t, y(t), y(t-\tau)), \\
y\left(t_{0}+\theta\right)=\varphi(\theta), \quad-\tau \leqslant \theta \leqslant 0 .
\end{gathered}
$$

Здесь $\tau>0, y(t): \mathbb{R} \rightarrow \mathbb{R}, f: \mathbb{R}^{3} \rightarrow \mathbb{R}, \varphi:[-\tau, 0] \rightarrow \mathbb{R}, t \geqslant t_{0}$.

Все известные численные методы интегрирования таких дифференциальных уравнений основаны на том, что функции, стоящие в правых частях уравнений, должны иметь смысл на интервале интегрирования. Численное решение задачи $(1),(2)$ с их помощью будет протекать успешно, если функция $f$, стоящая в правой части уравнения, является равномерно ограниченной. Ситуация будет ухудшаться при возрастании этой функции. Если же функция $f$ не ограничена, то решение такой задачи никаким численным методом получить невозможно.

Однако, уравнение (1), (2) в случае неограниченной функции, стоящей в правой части, может быть преобразовано в эквивалентную систему уравнений с ограниченными функциями, которую уже можно проинтегрировать численно. Более того, можно отыскивать решение задачи Коши, интегральная кривая которой является замкнутой.

Цель данного исследования заключается в том, чтобы получить для задачи (1), (2) такое преобразование.

2. Постановка и решение задачи. Рассмотрим задачу Коши (1) для нормальной системы обыкновенных дифференциальных уравнений с запаздьвающим аргументом. Здесь $\tau>0, y(t): \mathbb{R} \rightarrow \mathbb{R}^{n}, f: \mathbb{R}^{2 n+1} \rightarrow \mathbb{R}^{n}, t \in \mathbb{R}$. Начальные условия для задачи (1) примем в виде $(2)$, где заданная функция $\varphi:[-\tau, 0] \rightarrow \mathbb{R}^{n}, t_{0} \in \mathbb{R}$.

Пусть решение задачи $(1),(2)$ задается интегралом

$$
F(y, t)=0, \quad F: \mathbb{R}^{n+1} \rightarrow \mathbb{R}^{n},
$$


который определяет единственную гладкую интегральную кривую в $(n+1)$-мерном евклидовом пространстве $\mathbb{R}^{n+1}:\{y, t\}$ с началом в точке

$$
y=y_{0}, \quad t=t_{0} \text {. }
$$

Соотношения (3) можно рассматривать как систему нелинейных алгебраических или трансцендентных уравнений, содержащих параметр $t$, который, очевидно, является аргументом задачи (1), (2). Проблему построения кривой, определяемой уравнениями (3), будем изучать при помощи метода продолжения решения по параметру [2], [3].

Напомним основную идею этого метода, предложенного впервые, по-видимому, в [2].

Требуется построить кривую множества решений системы уравнений (3) с началом в точке (4). Эта кривая получается, если решать систему (3) при различных значениях параметра задачи $t \in\left[t_{0}, T\right]$, где значение $t=T$ соответствует концу кривой. Полагая, что $y_{i}(i=\overline{1, n})$ являются функциями параметра задачи $t$, уравнения продолжения строятся дифференцированием соотношений (3) по $t$ :

$$
\frac{\partial F}{\partial y} \frac{d y}{d t}+\frac{\partial F}{\partial t}=0
$$

Далее для осуществления процесса построения кривой множества решений уравнений (3) систему линейных уравнений (5) следует разрешить относительно производных $d y / d t$, т.е. привести ее к нормальной форме Коши. При этом получаем следующую задачу Коши:

$$
\frac{d y}{d t}=-\left(\frac{\partial F}{\partial y}\right)^{-1} \frac{\partial F}{\partial t}, \quad y\left(t_{0}\right)=y_{0},
$$

которую можно интегрировать любым численньм методом.

Очевидно, что при разрешении системы линейных относительно производных уравнений (5) могут возникнуть значительные вычислительные трудности, если матрица Якоби $\partial F / \partial y$ является плохо обусловленной, поэтому параметр задачи $t$, по которому проводится дифференцирование, не всегда может оказаться удачным. Следовательно, можно поставить вопрос о выборе наилучшего параметра продолжения решения системы (3) (см. [4], [5]), а значит, и наилучшего аргумента задачи (1), (2).

Пусть величины $t, y_{i}(i=\overline{1, n})$ являются функциями некоторого аргумента $\mu$, отсчитьваемого от точки (4), приращение которого в каждой точке интегральной кривой задачи (1), (2) представим в виде

$$
\Delta \mu=\sum_{i=1}^{n} \alpha_{i} \Delta y_{i}+\alpha_{n+1} \Delta t
$$

Здесь $\Delta t, \Delta y_{i}(i=\overline{1, n})$ - приращения соответствующих функций, $\alpha_{k}(k=\overline{1, n+1})-$ компоненты вектора $\alpha=\left(\alpha_{1}, \ldots, \alpha_{n+1}\right)^{T}$, задающего направление, в котором отсчитывается аргумент $\mu$.

Для того чтобы все направления были равноправными, вектор $\alpha$ должен быть единичным, т.е. удовлетворять равенству

$$
\alpha^{2}=\sum_{j=1}^{n+1} \alpha_{j}^{2}=1 .
$$


Правую часть равенства (6) можно рассматривать как скалярное произведение вектора $\alpha$ на вектор приращения функций. Придавая компонентам вектора $\alpha$ различные значения, можно рассмотреть все возможные параметры продолжения, а значит, и аргументы. Так, при $\alpha=(0, \ldots, 0,1)^{T}$ в качестве параметра-аргумента принимается переменная $t$.

Очевидно, что уравнения продолжения решения не могут быть получены дифференцированием соотношений (3) по параметру $\mu$, так как эти соотношения до решения задачи (1), (2) неизвестны.

Однако, уравнения продолжения могут быть получены другим способом.

Поскольку задача (1), (2) имеет единственную интегральную кривую, то системы уравнений (1) и (3) с учетом условий (2) и (4) задают одну и ту же кривую и, следовательно, в качестве уравнений продолжения решения может быть использована система (1). Поэтому уравнения продолжения решения получим, если равенство (6) разделим на $\Delta \mu$, устремив эту величину к 0 , а уравнения (1) перепишем с учетом равенства

$$
\frac{d y}{d t}=\frac{d y / d \mu}{d t / d \mu}
$$

При этом приходим к следующей системе уравнений продолжения:

$$
\begin{gathered}
\frac{d y}{d \mu}-f(t(\mu), y(\mu), y(t(\mu)-\tau)) \frac{d t}{d \mu}=0, \\
\sum_{i=1}^{n} \alpha_{i} \frac{d y_{i}}{d \mu}+\alpha_{n+1} \frac{d t}{d \mu}=1 .
\end{gathered}
$$

Очевидно, что кривая (3), являясь решением задачи $(1),(2)$, в то же время является интегральной кривой системы (8).

Для нахождения этой кривой систему (8) следует разрешить относительно производных. Успех такой операции зависит от обусловленности этой системы. Обусловленность же зависит от выбора параметра-аргумента $\mu$, которьй определяется вектором $\alpha$.

Полагаем, что система линейных уравнений является хорошо обусловленной, если малым изменениям элементов ее матрицы соответствует малое изменение решения системы уравнений.

В качестве меры обусловленности системы линейных уравнений примем [6] величину определителя этой системы $\Delta$, деленную на произведение квадратичных норм его строк $d$, которую обозначим через $D$. В силу неравенства Адамара для определителей абсолютная величина $D$ удовлетворяет условию $0 \leqslant|D| \leqslant 1$. Значение $D=0$ соответствует вырожденному случаю, а $|D|=1$ означает, что система уравнений является идеально обусловленной [6].

Назовем наилучшим такой параметр или такой аргумент, который доставляет линейной системе уравнений продолжения (8) наилучшую обусловленность, т.е. наибольшее абсолютное значение меры обусловленности $D$.

Легко видеть, что для системы уравнений (8) функция $\Delta$ задается следующим выражением:

$$
\Delta=\sum_{i=1}^{n} \alpha_{i} f_{i}+\alpha_{n+1}
$$


$\Phi$ ункция $d$ в силу условия (7) не зависит от компонент вектора $\alpha$, а значит, от выбора аргумента $\mu$ и вычисляется по формуле

$$
d=\prod_{i=1}^{n} \sqrt{1+f_{i}^{2}}
$$

Если систему уравнений (8) разрешить относительно производных, используя правило Крамера, то получаем нормальную систему дифференциальных уравнений вида

$$
\frac{d y}{d \mu}=\frac{f}{\Delta}, \quad \frac{d t}{d \mu}=\frac{1}{\Delta},
$$

которую следует интегрировать при начальных условиях

$$
y(0)=y_{0}=\varphi(0), \quad t(0)=t_{0}, \quad y\left(t_{0}+\theta\right)=\varphi(\theta), \quad-\tau \leqslant \theta \leqslant 0 .
$$

Здесь аргумент $\mu$ отсчитывается от начальной точки (4) задачи Коши (1), (2).

Основным результатом работы является следующая

ТЕоремА. Для того чтобы задачу Коши (1), (2) преобразовать к наилучиему аргументу, необходимо и достаточно выбрать в качестве такого аргумента длину дуги $\lambda$, вычисляемую вдоль интегральной кривой этой задачи. При этом преобразованная система уравнений будет иметь вид

$$
\begin{aligned}
& \frac{d y}{d \lambda}=\frac{f(t(\lambda), y(\lambda), y(t(\lambda)-\tau))}{\sqrt{1+\sum_{i=1}^{n} f_{i}^{2}(t(\lambda), y(\lambda), y(t(\lambda)-\tau))}}, \\
& \frac{d t}{d \lambda}=\frac{1}{\sqrt{1+\sum_{i=1}^{n} f_{i}^{2}(t(\lambda), y(\lambda), y(t(\lambda)-\tau))}} .
\end{aligned}
$$

Таким образом, если наилучший аргумент $\lambda$ отсчитьвать от начальной точки (4) задачи Коши (1), (2), то эта задача преобразуется в задачу (13), (12).

ДокАЗАТЕЛЬСтво. Необходимость. Для нахождения экстремума функции $D$ при условии (7) составим функцию Лагранжа

$$
L=\frac{1}{d}\left(\sum_{i=1}^{n} \alpha_{i} f_{i}+\alpha_{n+1}\right)+\gamma\left(1-\sum_{j=1}^{n+1} \alpha_{j}^{2}\right) .
$$

Здесь $\gamma$ - неопределенньй множитель Лагранжа.

Экстремум этой функции достигается при

$$
\frac{\partial L}{\partial \alpha_{k}}=\frac{f_{k}}{d}-2 \gamma \alpha_{k}=0, \quad k=\overline{1, n}, \quad \frac{\partial L}{\partial \alpha_{n+1}}=\frac{1}{d}-2 \gamma \alpha_{n+1}=0 .
$$

Эти соотношения позволяют выразить $\alpha_{k}$ через $\alpha_{n+1}$. Тогда из условия $(7)$ получаем

$$
\alpha_{n+1}=\frac{1}{\sqrt{1+\sum_{i=1}^{n} f_{i}^{2}}}, \quad \alpha_{k}=\frac{f_{k}}{\sqrt{1+\sum_{i=1}^{n} f_{i}^{2}}}, \quad k=\overline{1, n} .
$$


При этих значениях компонент вектора $\alpha$ определитель (9) равен $\Delta=\sqrt{1+\sum_{i=1}^{n} f_{i}^{2}}$, и преобразованная система (11) примет вид

$$
\frac{d y_{k}}{d \mu}=\frac{f_{k}}{\sqrt{1+\sum_{i=1}^{n} f_{i}^{2}}}, \quad k=\overline{1, n}, \quad \frac{d t}{d \mu}=\frac{1}{\sqrt{1+\sum_{i=1}^{n} f_{i}^{2}}} .
$$

Если правые части этой системы дифференциальных уравнений сравнить с выражениями для компонент вектора $\alpha(15)$, то приходим к равенствам

$$
\alpha_{k}=\frac{f_{k}}{\sqrt{1+\sum_{i=1}^{n} f_{i}^{2}}}=\frac{d y_{k}}{d \mu}, \quad k=\overline{1, n}, \quad \alpha_{n+1}=\frac{1}{\sqrt{1+\sum_{i=1}^{n} f_{i}^{2}}}=\frac{d t}{d \mu}
$$

которые показывают, что направление, в котором отсчитывается наилучший аргумент $\lambda$, должно быть касательным к интегральной кривой задачи $(1),(2)$.

С учетом равенств (16) последнее уравнение системы (8) можно записать в виде

$$
(d \mu)^{2}=\sum_{i=1}^{n}\left(d y_{i}\right)^{2}+(d t)^{2}
$$

откуда следует, что $d \mu=d \lambda=\sqrt{\sum_{i=1}^{n}\left(d y_{i}\right)^{2}+(d t)^{2}}$ является дифференциалом длины дуги $\lambda$ интегральной кривой задачи (1), (2). Если положить, что начальной точке этой задачи соответствует значение $\lambda=0$, то параметр $\lambda$ будет равен длине интегральной кривой, отсчитываемой от этой точки, и мы приходим к преобразованной задаче (13), (12).

Покажем, что при значениях компонент вектора $\alpha$, определяемых формулами (15), абсолютная величина функции $D=\Delta / d$ принимает наибольшее значение. В этом легко убедиться, учитывая, что знакоопределенность второго дифференциала функции Лагранжа $d^{2} L=-\gamma \sum_{j=1}^{n+1}\left(d \alpha_{j}\right)^{2}$ зависит от знака множителя Лагранжа $\gamma$. Знак же $\gamma$ совпадает со знаком определителя $\Delta(9)$. В самом деле, если $j$-е равенство (14) умножить на $\alpha_{j}$ и затем все их сложить, то с учетом условия (7) и выражения для $\Delta(9)$, получаем $\gamma=\Delta /(2 d)$.

Следовательно, при значениях $\alpha_{k}$, вычисляемых по формулам $(15)$, функция $|D|$, определяюшая меру обусловленности системы (8), принимает наибольшее значение, а это значит, что преобразованная система дифференциальных уравнений (8) будет наилучшим образом обусловленной. Необходимость доказана.

Достаточность. Выберем в качестве аргумента системы дифференциальных уравнений (1) длину дуги $\lambda$, вычисляемую вдоль интегральной кривой задачи $(1),(2)$. Вектор $\tau$, касательньй к этой кривой, будет равен $\tau=\left(y_{1, \lambda}, \ldots, y_{n, \lambda}, t_{\lambda}\right)^{T}$. Как отмечалось ранее, смысл единичного вектора $\alpha=\left(\alpha_{1}, \ldots, \alpha_{n+1}\right)^{T}$ заключается в том, что он определяет направление выбора аргумента задачи $(1),(2)$. Следовательно, векторы $\alpha$ и $\tau$ должны быть коллинеарными. Однако, они не только коллинеарны, но и равны, так как вектор $\tau$, как и $\alpha$, является единичным. В самом деле, дифференциал длины интегральной кривой задачи (1), (2) должен удовлетворять равенству (17), в котором следует положить $d \mu=d \lambda$. Если обе части этого равенства разделить на $(d \lambda)^{2}$, то получим

$$
\tau^{2}=\sum_{k=1}^{n} y_{k, \lambda}^{2}+t_{\lambda}^{2}=1
$$


Из равенства векторов следует равенство компонент

$$
\alpha_{k}=\frac{d y_{k}}{d \lambda}, \quad k=\overline{1, n}, \quad \alpha_{n+1}=\frac{d t}{d \lambda} .
$$

Компоненты же $t_{\lambda}, y_{k, \lambda}(k=\overline{1, n})$ должны удовлетворять системе дифференциальных уравнений (11), которую с учетом равенств (18), (9) можно представить в виде

$$
\frac{d y_{k}}{d \lambda}=\frac{f_{k}}{\sum_{i=1}^{n} y_{i, \lambda} f_{i}+t_{\lambda}}, \quad \frac{d t}{d \lambda}=\frac{1}{\sum_{i=1}^{n} y_{i, \lambda} f_{i}+t_{\lambda}}, \quad k=\overline{1, n} .
$$

Если каждое $j$-е уравнение этой системы умножить на $f_{j}(j=\overline{1, n+1})$, полагая $f_{n+1}=1$, и сложить полученные соотношения, то выражение, стоящее в знаменателе правой части системы (19), примет вид

$$
\sum_{i=1}^{n} y_{i, \lambda} f_{i}+t_{\lambda}=\sqrt{1+\sum_{i=1}^{n} f_{i}^{2}}
$$

и система дифференциальных уравнений в точности совпадет с преобразованной системой (13).

Тогда с учетом равенств $(18)$ функции $\alpha_{j}(j=\overline{1, n+1})$, будут выгисляться по формулам (15), которые, как установлено выше, доставляют наибольшее значение функции $|D|$ и определяют наилучшую обусловленность системы уравнений (8). Теорема доказана.

ЗАМЕЧАНИЕ 1. Наилучшей обусловленности системы уравнений (8) будет соответствовать наибольшее абсолютное значение меры обусловленности $D=\Delta / d$, а значит, в силу того, что функция $d(10)$ не зависит от вектора $\alpha$, и наибольшее абсолютное значение определителя $\Delta(9)$. В этом случае абсолютные значения функций, стоящих в правой части системы (11), принимают свои наименьшие возможные значения. Поэтому можно дать другое эквивалентное определение наилучшего аргумента задачи (1), (2): наилучшим назьвается такой аргумент, при переходе к которому функции, стояшие в правой части преобразованной системы уравнений (11), принимают свои наименьшие абсолютные значения.

Таким образом, для системы уравнений, преобразованной к наилучшему аргументу, малому приращению аргумента соответствует наименьшее прирашение искомых функций.

ЗАмЕчАниЕ 2. Как видно из последнего уравнения системы (13), выбор положительного знака перед корнем соответствует перемещению вдоль интегральной кривой в сторону возрастания функции $t$. Очевидно, что знак “-” будет соответствовать перемещению в противоположном направлении.

ЗАмЕчаниЕ 3. Преобразованная задача Коши (13), (12) выгодно отличается от задачи Коши (1), (2). В самом деле, абсолютные значения функций, стоящих в правой части системы уравнений (13), не превосходят единицы, более того, квадратичная норма правой части этой системы всегда равна единище. Это во многих случаях позволяет преобразовать уравнения с неограниченньми функциями, стоящими в правой части, в систему уравнений с ограниченньми функциями, что может оказаться полезным как 
при численном, так и при качественном исследовании задачи. Такой подход позволяет также решать дифференциальные уравнения, интегральные кривые которых имеют предельные точки или даже являются замкнутыми.

Рассмотренное преобразование для системы обыкновенных дифференциальных уравнений без запаздьвания впервые было предложено в [7], а в [8] показано, что это преобразование улучшает вычислительную ситуацию в случае жестких систем обыкновенных дифференциальных уравнений. Это нашло подтверждение при решении жестких задач, рассмотренных в [5], [8].

Из исследований [8] также следует, что эффективность предложенного подхода повышается при решении обыкновенньх дифференциальных уравнений, у которьх функции, стоящие в правых частях, принимают большие значения.

В заключение обратим внимание на некоторые особенности предложенного подхода.

1. Линейные уравнения преобразуются в нелинейные, что при численном решении задачи, вообще говоря, не является большим недостатком.

2. Увеличивается на единицу порядок интегрируемой системы уравнений. Однако, значение этого обстоятельства будет уменьшаться при увеличении порядка системы.

3. Преобразование не уменьшает интервал интегрирования. Однако, в [9] на примере модельного обыкновенного дифференциального уравнения без запаздьвания показано, что, удовлетворяя условиям устойчивости явного метода Эйлера, продвигаясь по наилучшему аргументу $\lambda$ преобразованной задачи, можно попасть в конечную точку интегральной кривой за меньшее число шагов, чем двигаясь по аргументу исходной задачи $t$.

\section{СПИСОК ЦИТИРОВАННОЙ ЛИТЕРАТУРЫ}

[1] Хейл Дж. Теория функционально-дифференциальных уравнений. М.: Мир, 1984.

[2] Давиденко Д. Ф. Об одном новом методе численного решения систем нелинейных уравнений // Докл. АН СССР. 1953. Т. 88. №4. С. 601-602.

[3] Григолюк Э. И., Шалашилин В.И. Проблемы нелинейного деформирования. М.: Наука, 1988.

[4] Шалашилин В.И., Кузнецов Е.Б. Наилучший параметр продолжения решения // Докл. РАН. 1994. Т. 334. № 5. С. 566-568.

[5] Кузнецов Е. Б., Шалашилин В. И. Задача Коши как задача продолжения по наилучшему параметру // Дифференц. уравнения. 1994. Т. 30. №6. С. 964-971.

[6] Ортега Дж., Пул У. Введение в численные методы решения дифференциальных уравнений. М.: Наука, 1986.

[7] Шалашилин В. И., Кузнецов Е. Б. Задача Коши для нелинейно деформируемых систем как задача продолжения решения по параметру // Докл. РАН. 1993. Т. 329. № 4. С. 426-428.

[8] Кузнецов Е. Б., Шалашилин В.И.Задача Коши как задача продолжения решения по параметру // ЖВМиМФ. 1993. Т. 33. № 12. С. 1792-1805.

[9] Кузнецов Е. Б., Шалашилин В.И.Задача Коши для механических систем с конечным числом степеней свободы как задача продолжения по наилучшему параметру // ПММ. 1994. T. 58. №6. С. 14-21. 